\title{
A cinética do chumbo no organismo humano e sua importância para a saúde
}

\author{
Lead kinetics in human body and its \\ significance to health
}

Fátima Ramos Moreira ${ }^{1}$ Josino Costa Moreira 1

\footnotetext{
1 Centro de Estudos da Saúde do Trabalhador e Ecologia Humana (CESTEH), Escola Nacional de Saúde Pública (ENSP), Fiocruz.

Av. Leopoldo Bulhões 1480, Manguinhos, 21041-210, Rio de Janeiro RJ. fmoreira@ensp.fiocruz.br
}

\begin{abstract}
Lead is one of the most common pollutants in the environment. It is considered toxic to the human beings and animals and without any physiological known function in the organism. Lead toxic effects can affect almost all organs and systems of the body. Lead enters the body primarily through inhalation or ingestion, being directly absorbed, distributed, and excreted. The primary sites for inorganic lead absorption are gastrointestinal and respiratory tract. Once absorbed, lead is found in blood, soft tissues and mineralizing systems. Approximately 90\% of the total body burden of lead is found in bones, body's major storage site. Around $5 \%$ of lead in blood is in plasma, representing the labile and biologically active lead fraction, able to pass the cells membranes and cause toxic effects. The absorbed lead is excreted mainly through urine and feces. Thus the knowledge of the lead kinetics is important to a greater understanding on lead toxicity, since the risks of health adverse effects are related to the total body burden of lead.
\end{abstract}

Key words Lead, Kinetics, Bone, Plasma
Resumo O chumbo é um dos contaminantes ambientais mais comuns, tóxico para os homens e animais, e sem nenhuma função fisiológica conhecida no organismo. Seus efeitos nocivos podem afetar praticamente todos os órgãos e sistemas do organismo humano. O chumbo entra no corpo principalmente por inalação ou ingestão, sendo diretamente absorvido, distribuído e excretado. Os tratos gastrointestinal e respiratório são os principais sitios de absorção do chumbo que, uma vez absorvido, é encontrado no sangue, tecidos moles e mineralizados. Cerca de $90 \%$ do chumbo corpóreo se armazena nos ossos, principal depósito do metal no corpo. Aproximadamente 5\% da concentração do chumbo no sangue se situa no plasma, representando a fração lábil e biologicamente ativa do chumbo, capaz de cruzar as membranas celulares e causar seus efeitos tóxicos. $O$ chumbo absorvido é excretado principalmente pela urina e fezes. Assim, o conhecimento da cinética do chumbo é importante para maior compreensão da toxicidade deste metal, uma vez que os riscos de efeitos adversos à saúde estão relacionados com o conteúdo corpóreo total do chumbo. Palavras-chave Chumbo, Cinética, Osso, Plasma 


\section{Introdução}

O chumbo $(\mathrm{Pb})$ é um elemento de ocorrência natural, amplamente utilizado há milhares de anos. Atualmente, é um dos contaminantes mais comuns do ambiente, devido às inúmeras atividades industriais que favorecem a sua grande distribuição. Assim, todos os seres humanos têm chumbo em seus organismos como resultado da exposição às fontes exógenas (Saryan e Zenz, 1994). No entanto, este metal não possui nenhuma função fisiológica conhecida no organismo, e seus efeitos tóxicos sobre os homens e animais já são conhecidos há muito tempo por afetarem praticamente todos os órgãos e sistemas do corpo humano (Xie et al., 1998).

Nos últimos tempos, a cinética, ou seja, a interação contínua entre a absorção, distribuição, armazenamento e eliminação do chumbo vem sendo exaustivamente estudada em homens e animais. Esses processos fisiológicos podem ser influenciados por vários fatores endógenos (por exemplo, constituição genética, fatores antropométricos e estado de saúde) e exógenos, tais como carga de trabalho, exposição simultânea a várias substâncias, drogas, álcool e fumo. O conhecimento desses fatores é essencial para a seleção dos indicadores biológicos apropriados, meio biológico a ser analisado e tempo de amostragem, e para a interpretação do resultado (WHO, 1996). Entretanto, apesar dos numerosos estudos, muitos aspectos do metabolismo normal do chumbo ainda são pouco entendidos. O que se conhece é, na maioria das vezes, derivado das respostas dos níveis de chumbo em tecidos e fluidos do organismo às mudanças na exposição e entrada de chumbo ao longo do tempo (Rabinowitz et al., 1976).

A maior parte do chumbo entra no organismo humano pelas vias respiratória e gastrointestinal. Após absorção, o chumbo pode ser encontrado no sangue, tecidos moles e mineralizados (ATSDR, 1999). O sítio primário de armazenamento de $\mathrm{Pb}$ no organismo é o osso, que contém aproximadamente $95 \%$ do conteúdo corpóreo total do metal em adultos, porém o significado toxicológico deste fato só foi entendido recentemente (Sanín et al., 1998). Historicamente considerado como um depósito inerte de chumbo, hoje o esqueleto é reconhecido como sendo tão importante quanto as influências da exposição, absorção e eliminação para o comportamento toxicocinético do chumbo no organismo humano (Smith et al., 1996).
Inúmeros estudos têm fornecido evidências de que a mobilização de $\mathrm{Pb}$ dos ossos para o sangue é intensificada durante os períodos de maior liberação óssea tais como crescimento rápido na infância, gravidez, lactação, menopausa, desequilíbrios hormonais, entre outros (Smith et al., 1996). Uma vez na corrente sangüínea, os processos cinéticos de absorção, trocas com outros compartimentos do organismo e de eliminação determinam o teor de chumbo no sangue (Hryhorczuk et al., 1985).

Embora a concentração de chumbo no sangue $(\mathrm{Pb}-\mathrm{S})$ seja aceita como indicador de exposição total ao metal, dados recentes sugerem que $\mathrm{Pb}-\mathrm{S}$ não representa adequadamente os níveis desse elemento nos ossos e nem no plasma (fração toxicologicamente mais ativa e biodisponível para a maioria dos órgãos), e que os estoques de chumbo nos ossos podem influenciar de maneira independente a sua concentração no plasma. Entretanto, algumas informações adicionais ainda são necessárias sobre o quanto de chumbo é mobilizado do osso em indivíduos expostos (Tsaih et al., 1999). O comportamento cinético e a toxicidade do chumbo em humanos são determinados grandemente por meio de mecanismos pelos quais o metal é trocado entre o plasma e as superfícies ósseas, processos de crescimento e ressorção do osso, $\mathrm{e}$ processos de troca heteroiônica nos rins e intestinos (ATSDR, 1999).

A fisiologia do chumbo em humanos normalmente desperta um interesse considerável, gerado pela toxicidade potencial desse elemento. O chumbo inorgânico pode afetar uma série de sistemas, cuja grandeza das manifestações clínicas dependerá da intensidade, do tempo de exposição e da sensibilidade individual. A toxicidade está relacionada principalmente aos sistemas nervoso e hematopoiético. Entretanto, podem ocorrer efeitos renais, cardiovasculares e na reprodução, não existindo, porém, evidências adequadas de carcinogenicidade em humanos. Alguns estudos têm correlacionado efeitos adversos à saúde com níveis cada vez mais baixos de chumbo no sangue, até mesmo em concentrações menores do que $10 \mu \mathrm{g} \cdot \mathrm{dL}^{-1}$, principalmente em crianças (ATSDR, 1992).

Nos países desenvolvidos, o controle das fontes de poluição de chumbo vem sendo implementado gradativamente, com uma intensa atuação dos órgãos ambientais e da saúde pública. No Brasil, o controle é praticamente inexistente, devido à falta de dados sobre a real exposição da população. Por isso, são necessários 
estudos que forneçam as informações indispensáveis para melhor conhecimento da nossa realidade e, desse modo, subsidiar os órgãos de saúde pública e meio ambiente nas ações de controle.

Esta revisão tem como principal objetivo mostrar o comportamento cinético do chumbo no organismo humano, apresentando algumas das muitas pesquisas realizadas nas diferentes etapas da toxicocinética deste metal e os resultados obtidos. E, então, permitir uma visão geral acerca do atual estágio de conhecimento sobre o assunto e mostrar a importância da total compreensão da cinética do chumbo, que leva a importantes alterações biológicas, para a avaliação da exposição, direcionando assim para a necessidade de uma maior informação sobre os efeitos deste elemento no organismo humano.

\section{Absorção}

No organismo humano, o chumbo não é metabolizado, e sim, complexado por macromoléculas, sendo diretamente absorvido, distribuído e excretado. Os compostos de chumbo inorgânico entram no organismo por inalação (rota mais importante na exposição ocupacional) ou ingestão (via predominante para a população em geral). Somente os compostos orgânicos de chumbo são capazes de penetrar através da pele íntegra. Os compostos de chumbo tetra alquila (chumbo tetra etila, etc.), por exemplo, são absorvidos rapidamente pelos pulmões, trato gastrointestinal e também pela pele (Tsalev e Zaprianov, 1985).

A absorção do chumbo no organismo é influenciada pela rota de exposição, espécie química formada, tamanho da partícula (no caso de particulado), solubilidade em água, e variações individuais fisiológicas e patológicas. A absorção do chumbo no sangue pode ser superior a $50 \%$ da dose inalada/ingerida para gases de exaustão e sais altamente solúveis, assim como para fumantes e pessoas com doenças das vias respiratórias superiores, que têm a atividade ciliar prejudicada, favorecendo assim uma maior deposição das partículas de chumbo no trato respiratório (Saryan e Zenz, 1994).

A absorção do chumbo no trato gastrointestinal varia de $2 \%$ a $16 \%$ se ingerido com refeição, mas pode chegar a $60-80 \%$, quando administrado em jejum. Mulheres grávidas e crianças absorvem $45 \%$ a $50 \%$ do chumbo presente na dieta. Esta absorção ocorre originalmente no duodeno por mecanismos ainda indefinidos, podendo, porém, envolver transporte ativo e/ou difusão trans ou paracelular, tanto do chumbo ionizado quanto dos complexos inorgânicos ou orgânicos deste elemento (ATSDR, 1999). No entanto, os resultados de um estudo publicado por Gulson et al. (1997b) sugerem que a absorção do chumbo ingerido por crianças de 6 a 11 anos é comparável com os padrões de absorção observados em mulheres na faixa de 29 a 37 anos de idade.

A absorção pelo trato gastrointestinal depende mais de fatores nutricionais tais como ingestão de cálcio $(\mathrm{Ca})$, ferro $(\mathrm{Fe})$, fósforo $(\mathrm{P})$ e proteínas, do que da solubilidade dos compostos de chumbo, devido à acidez do estômago. Sabe-se que um baixo teor de $\mathrm{Ca}$ ou Fe na dieta aumenta a absorção do $\mathrm{Pb}$. O mesmo é verdadeiro para uma alimentação deficiente em $\mathrm{P}$ e proteínas. A absorção do chumbo pela mucosa intestinal possivelmente envolve um mecanismo de competição com relação ao cálcio (IPCS, 1995). Os resultados encontrados para as relações entre os fatores nutricionais e acumulação do $\mathrm{Pb}$ no organismo de 747 homens, com idades entre 49 e 93 anos, sugerem que a baixa ingestão diária de vitamina $\mathrm{D}$ pode aumentar a acumulação do $\mathrm{Pb}$ nos ossos, enquanto que menor ingestão diária de vitamina $\mathrm{C}$ e ferro pode aumentar os níveis de $\mathrm{Pb}$ no sangue. $\mathrm{Um}$ maior influxo de cálcio foi associado com níveis mais baixos de chumbo ósseo, porém essa relação se tornou insignificante após ajuste para a vitamina D (Cheng, 1998).

A concentração média de chumbo no sangue de camponesas suplementadas com 940mg Ca foi significativamente menor do que o nível médio deste metal encontrado no sangue das mulheres que ingeriram metade deste teor, sugerindo que a ingestão adequada de cálcio pode ser uma das medidas preventivas para a redução da absorção do chumbo (Kostial et al., 1991). Sargent e colaboradores (1999) perceberam que um efeito significativo da suplementação de cálcio nas fórmulas infantis sobre a concentração de chumbo no sangue aconteceu somente nos 4 primeiros meses do acompanhamento. Com isto, não puderam concluir que o suplemento de glicerofosfato de cálcio evitou a absorção do chumbo em 103 crianças com idade entre 3,5 e 6 meses durante os 9 meses de duração do ensaio.

Kristal-Boneh e colegas (1998) avaliaram os níveis de $\mathrm{Pb}-\mathrm{S}$ e Ca ingerido na dieta em 146 
trabalhadores. A média de cálcio ingerido era $775 \pm 370 \mathrm{mg} /$ dia nos indivíduos não-expostos e $858 \pm 423 \mathrm{mg} / \mathrm{dia}$ nos expostos, e concluíram que a quantidade de cálcio na dieta não influencia os níveis de chumbo no sangue. Resultados similares encontraram Morris e seus colaboradores (1990), em que a suplementação de $1 \mathrm{~g}$ Ca por dia, durante 12 semanas, não alterou significativamente os níveis de $\mathrm{Pb}$ no sangue e protoporfirina eritrocitária na população em geral.

Em estudos realizados com pintos, Fullmer (1995) investigou os efeitos associados aos níveis de cálcio e de chumbo da dieta sobre a função endócrina da vitamina $\mathrm{D}$ e verificou que a ingestão de chumbo pode resultar no aumento ou diminuição da concentração do hormônio da vitamina $\mathrm{D}$ circulante, levando a respostas intestinais dependentes do teor de cálcio na dieta e do tempo de exposição ao chumbo, fornecendo possíveis explicações para as conflitantes observações com respeito às interações chumbo-cálcio. Em outro experimento (1997), foram pesquisadas as interações entre o cálcio da dieta e o chumbo que influenciam os níveis do hormônio da vitamina $\mathrm{D}$ no soro, a absorção intestinal destes metais e a retenção do chumbo no organismo. Este estudo mostrou perfis de resposta diferentes para a absorção do $\mathrm{Ca} e$ do $\mathrm{Pb}$, sugerindo que o mecanismo de transporte intestinal dos dois cátions pode ser distinto.

Durante a gravidez, a absorção intestinal pode aumentar devido à maior mobilização do chumbo dos ossos, e contribuir para a elevação da concentração do metal no sangue observada no último trimestre da gestação (ATSDR, 1992). De acordo com Rothenberg e colegas (1994), a mobilização do chumbo do osso aumenta a absorção intestinal, explicando parte da tendência ascendente de $\mathrm{Pb}-\mathrm{S}$ na segunda metade da gravidez de moradoras do México. Chegaram a essa conclusão após a análise de variância revelar uma redução significativa na média do $\mathrm{Pb}-\mathrm{S}$ da $12 \mathrm{a}\left(7,0 \mu \mathrm{g} \cdot \mathrm{dL}^{-1}\right)$ para a 20 a semana $\left(1,1 \mu \mathrm{g} \cdot \mathrm{dL}^{-1}\right)$ e aumentos significativos na média de $\mathrm{Pb}-\mathrm{S}$ da 20 a semana até o parto $(1,6$ $\left.\mu \mathrm{g} . \mathrm{dL}^{-1}\right)$. Em outra pesquisa, os dados encontrados indicam que a mobilização dos depósitos de longo prazo (ossos, por exemplo) contribui significativamente para os níveis de chumbo no sangue durante o último trimestre da gestação e excede a troca normal de chumbo dos ossos observada fora de gravidez (Gulson et al., 1997a). Usando isótopos estáveis de $\mathrm{Pb}$ de alta precisão, Gulson e colaboradores mostraram que o $\mathrm{Pb}$ é mobilizado do esqueleto materno durante a gravidez e período pós-parto. As liberações observadas sugerem uma elevada troca óssea, maior do que $10 \%$, e possivelmente maior do que $30 \%$ em algumas mulheres (Gulson et al., 1999c).

Johnson (2001) propõe que o mecanismo pelo qual uma elevada ingestão de cálcio impede o aumento do chumbo no sangue materno induzido pela gravidez, pode envolver uma diminuição da absorção de chumbo no intestino ou da ressorção do osso materno, reduzindo assim a concentração do metal no sangue materno e, conseqüentemente, limitando a acumulação de chumbo no feto. Um estudo conduzido em ratas grávidas demonstrou que a adição de Ca na dieta poderia reduzir a transferência do chumbo, proveniente de exposições maternas anteriores à gestação, para o feto (Han et al., 2000). Entretanto, os resultados encontrados por Rothenberg e colegas (2000) sugerem que fontes exógenas e reguladoras do nível deste metal no sangue, tais como o uso de cerâmica vitrificada ou de cálcio na dieta, só controlam a exposição durante e após a gravidez. As fontes endógenas resultantes de exposições passadas continuariam a influenciar a concentração de $\mathrm{Pb}$ no sangue.

Numa investigação realizada por Baghurst e seus colaboradores (1987) com mulheres grávidas expostas ambientalmente, a baixa condição socioeconômica, o hábito de fumar e dieta pobre em cálcio foram alguns dos fatores associados com uma concentração de chumbo mais elevada no sangue. Estes pesquisadores também relatam que menores níveis do metal foram observados em mulheres que complementavam a dieta com ferro e ácido fólico.

A contaminação dos suplementos de cálcio com chumbo tem gerado muitas discussões, uma vez que a presença do metal pode contribuir para a elevação do $\mathrm{Pb}-\mathrm{S}$ e representar um problema de saúde pública facilmente evitável, conforme concluíram Ross e colaboradores (2000). Entretanto, os resultados de um estudo conduzido por Gulson e colegas (2001a) mostraram que não houve aumento perceptível na concentração de $\mathrm{Pb}$-S durante o consumo de suplemento de cálcio, porém mudanças significativas aconteceram na composição isotópica do chumbo no sangue, proveniente do suplemento de carbonato de cálcio, indicando uma contribuição da dieta para o Pb-S.

As crianças são mais sensíveis aos efeitos de chumbo mesmo em baixos níveis e o uso desses suplementos pode aumentar o risco à saú- 
de, particularmente naquelas com intolerância ao leite e seus derivados, e que suprem suas necessidades de cálcio com estas fórmulas. Porém, menos de $20 \%$ das 70 marcas de suplementos de cálcio pesquisadas por Bourgoin e colaboradores (1993) apresentaram níveis de chumbo comparáveis ou menores do que aqueles relatados para o leite de vaca $(25 \mu \mathrm{g} \mathrm{Pb} / \mathrm{kg}$ de peso do corpo). Já Scelfo e Flegal (2000) analisaram 136 marcas de suplementos, sintéticos, refinados e derivados de fontes naturais, e concluíram que dois terços deles ultrapassaram os critérios da Califórnia (1999) para níveis aceitáveis de chumbo em produtos para consumidores $(1,5 \mu \mathrm{g} \mathrm{Pb} /$ dose diária de $\mathrm{Ca})$.

\section{Distribuição}

A distribuição do chumbo pelo organismo depende de sua taxa de transferência da corrente sangüínea para os diferentes órgãos e tecidos. Entre crianças e adultos, existem inúmeras diferenças relacionadas à idade. Uma vez absorvido, o chumbo se distribui entre o sangue, os tecidos moles (rins, medula óssea, fígado e cérebro) e os tecidos mineralizados (ossos e dentes) (ATSDR, 1992).

Vários modelos cinéticos têm sido propostos para explicar a distribuição do chumbo no organismo. Rabinowitz e colaboradores (1976) sugeriram um modelo de três compartimentos (Figura 1) no qual o primeiro seria o sangue, que estaria em comunicação direta com os compartimentos dois e três. O segundo reservatório seria composto dos tecidos moles e o terceiro consistiria principalmente dos ossos, que conteria elevados níveis do chumbo corpóreo. Os tempos de meia-vida do $\mathrm{Pb}$ nos três compartimentos são bastante diferentes, sendo estimado em 36 dias para o sangue, 40 dias para os tecidos moles e 27 anos para os ossos, de acordo com este modelo. Semelhantemente, Nilsson e colegas (1991) concluíram que o esqueleto representa o compartimento em que a cinética de eliminação é a mais lenta (principalmente o osso cortical), enquanto que a mais rápida é observada no sangue e em alguns tecidos moles. Sugerem ainda um componente intermediário que, pelo menos parcialmente, corresponderia ao osso trabecular, que apresenta uma cinética diferenciada do osso cortical. Também um modelo cinético simples, de dois compartimentos (osso e sangue), é apresentado por Rabinowitz (1991), em que propõe valores numéricos para as alterações dos níveis de $\mathrm{Pb}-\mathrm{S}$, decorrentes das mudanças nas taxas de mobilização, variáveis com a idade e saúde. Duas propostas são oferecidas para quantificar a troca de chumbo, uma envolve o estudo de pessoas com exposição passada conhecida, e a outra abrange isótopos estáveis e sujeitos que se mudam de uma região geográfica para outra.

Informações adicionais sobre a biocinética do chumbo e exposição ao metal permitiram refinamentos e expansões dos modelos propostos anteriormente. Em especial, três modelos farmacocinéticos para adultos e crianças estão sendo normalmente considerados para ampla aplicação: o de O'Flaherty, o biocinético e de exposição integrados (Integrated Exposure Uptake and Biokinetic Model - IEUBK) e o de Leggett. Dos três, somente aquele proposto por O'Flaherty (Figura 2) usa parâmetros fisiologicamente consistentes para descrever o volume, a composição e a atividade metabólica do sangue e tecidos que determinam a distribuição do $\mathrm{Pb}$ no organismo humano. Os outros dois modelos são multicompartimentais clássicos, cujas taxas de transferência específicas da idade estão baseadas em dados cinéticos de estudos em animais e humanos, e podem não ter correlações fisiológicas precisas (ATSDR, 1999).

Um modelo de absorção e distribuição do chumbo baseado fisiologicamente, desenvolvido e validado para adultos numa ampla faixa de exposição e diversas fontes, foi modificado para incorporar informação adicional sobre a dependência da idade na taxa de formação do osso e considerar o local da atividade de modelagem do osso crescente com a idade (O'Flaherty, 1995). Como o metabolismo do osso é o centro do modelo de O'Flaherty, sua característica central é a curva de crescimento, que relaciona peso do corpo com idade. Os volumes dos ossos e órgãos, e funções fisiológicas são expressos como função do peso do corpo baseado em medidas feitas em humanos. As taxas de formação e ressorção do osso são estimadas a partir de estudos sobre a cinética do Ca marcado também realizados com humanos, sendo que os ossos cortical e trabecular são modelados separadamente, considerando as respectivas razões entre superfície e volume (O'Flaherty, 1998). Fleming e colaboradores (1999) selecionaram um grupo de trabalhadores de uma fundição de chumbo para avaliar o modelo de O'Flaherty e os resultados para essa população foram consistentes com a hipótese de que um polimorfismo na enzima ácido delta aminole- 
Figura 1

Modelo para o metabolismo do chumbo de acordo com Rabinowitz et al. (1976).

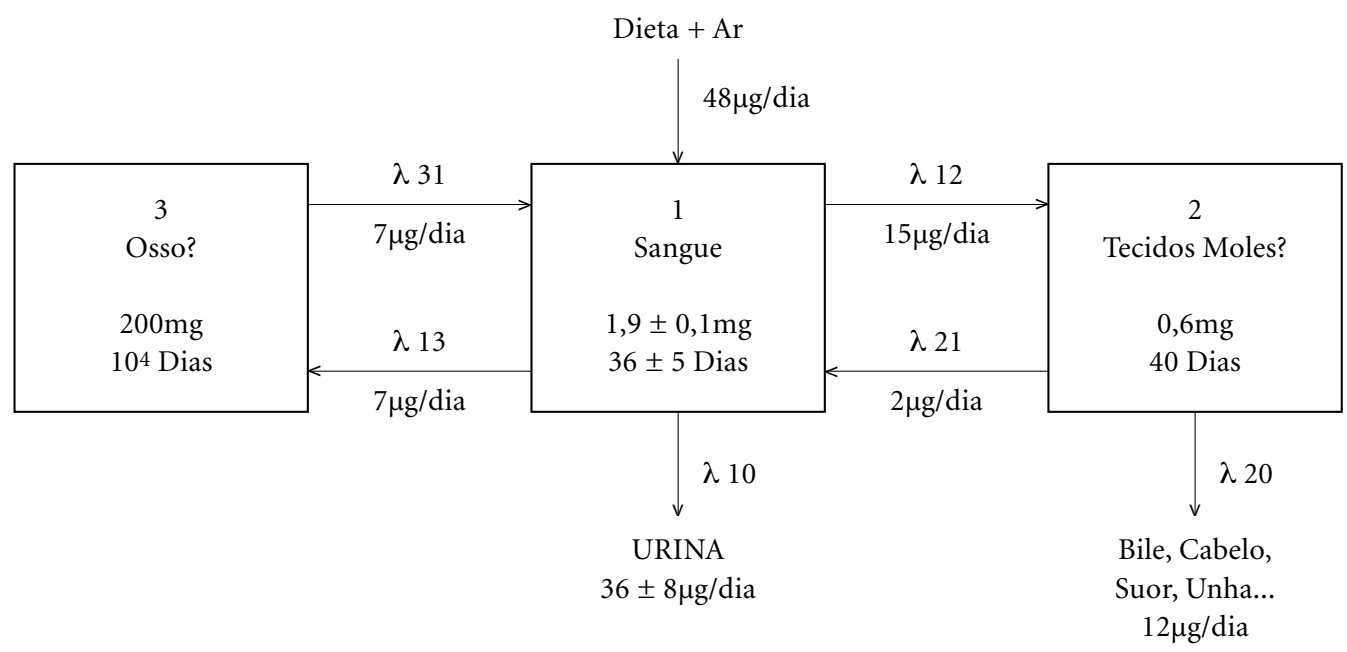

vulínico desidratase (ALAD) modifica a cinética do chumbo em humanos.

Em adultos, os ossos contêm cerca de $90 \%$ a $95 \%$ do conteúdo corpóreo total de chumbo, enquanto que $80 \%$ a $95 \%$ são encontrados nas crianças (Hu, 1998). Embora a concentração de chumbo em sangue seja menor do que $2 \%$ do seu total no organismo, de $90 \%$ a $99,8 \%$ do metal estão ligados à membrana e a frações de proteínas (principalmente à hemoglobina, $\mathrm{e}$ outras proteínas de baixo peso molecular) das células vermelhas (Goyer, 1991).

Bergdahl e colaboradores (1997a) concluíram que a principal proteína de ligação ao chumbo era a ácido delta aminolevulínico desidratase (ALAD), sugerindo que a noção anterior de que, nos eritrócitos, o $\mathrm{Pb}$ se ligava primeiramente à hemoglobina deveria ser revista. Nas proteínas obtidas dos eritrócitos lisados, além da maior afinidade do Pb pela ALAD, também foram identificadas ligações do chumbo com uma proteína de massa molecular de $45 \mathrm{kDa}$ e com outra de massa menor do que 10 $\mathrm{kDa}$. No entanto, nenhum chumbo ligado à hemoglobina foi detectado. Assim, um novo modelo foi proposto, baseado nesses três componentes e em mais um quarto, que representaria o $\mathrm{Pb}$ não recuperado (Bergdahl et al., 1998). Xie e seus colegas (1998) encontraram semelhança no padrão de distribuição das proteínas para os experimentos in vivo e in vitro. Em ambos os casos, uma maior concentração de chumbo foi determinada na região correspondente à massa molecular do ALAD e de um outro pico, eluído após a fração da hemoglobina, na região de massa estimada entre 30 e $50 \mathrm{kDa}$.

Os modelos propostos para representar a cinética do $\mathrm{Pb}$ normalmente supõem que o seu transporte pelo corpo é comandado pelo componente plasma do sangue total (O'Flaherty, 1995). Porém, em concentrações elevadas de $\mathrm{Pb}-\mathrm{S}$, há uma saturação gradual dos sítios de ligação nos eritrócitos e uma maior quantidade deste elemento se torna biologicamente disponível. Este mecanismo é consistente com os resultados encontrados por Fleming e colaboradores (1997), em que os trabalhadores mais antigos de uma fundição apresentaram concentrações de chumbo no sangue relativamente altas, podendo freqüentemente ter excedido tal ponto de saturação, enquanto que trabalhadores empregados há menos tempo teriam níveis de $\mathrm{Pb}-\mathrm{S}$ abaixo deste ponto.

Resultados de estudos isotópicos sobre a liberação do $\mathrm{Pb}$ do osso para a circulação mostraram que $40 \%$ a $70 \%$ do chumbo presente no sangue de sujeitos expostos ambientalmente (Smith et al., 1996) e de mulheres adultas (Gulson et al., 1995) são provenientes do esqueleto. O osso influencia a concentração do chumbo 
Figura 2

Compartimentos e caminhos de troca do chumbo no modelo de O'Flaherty (O'Flaherty, 1998).

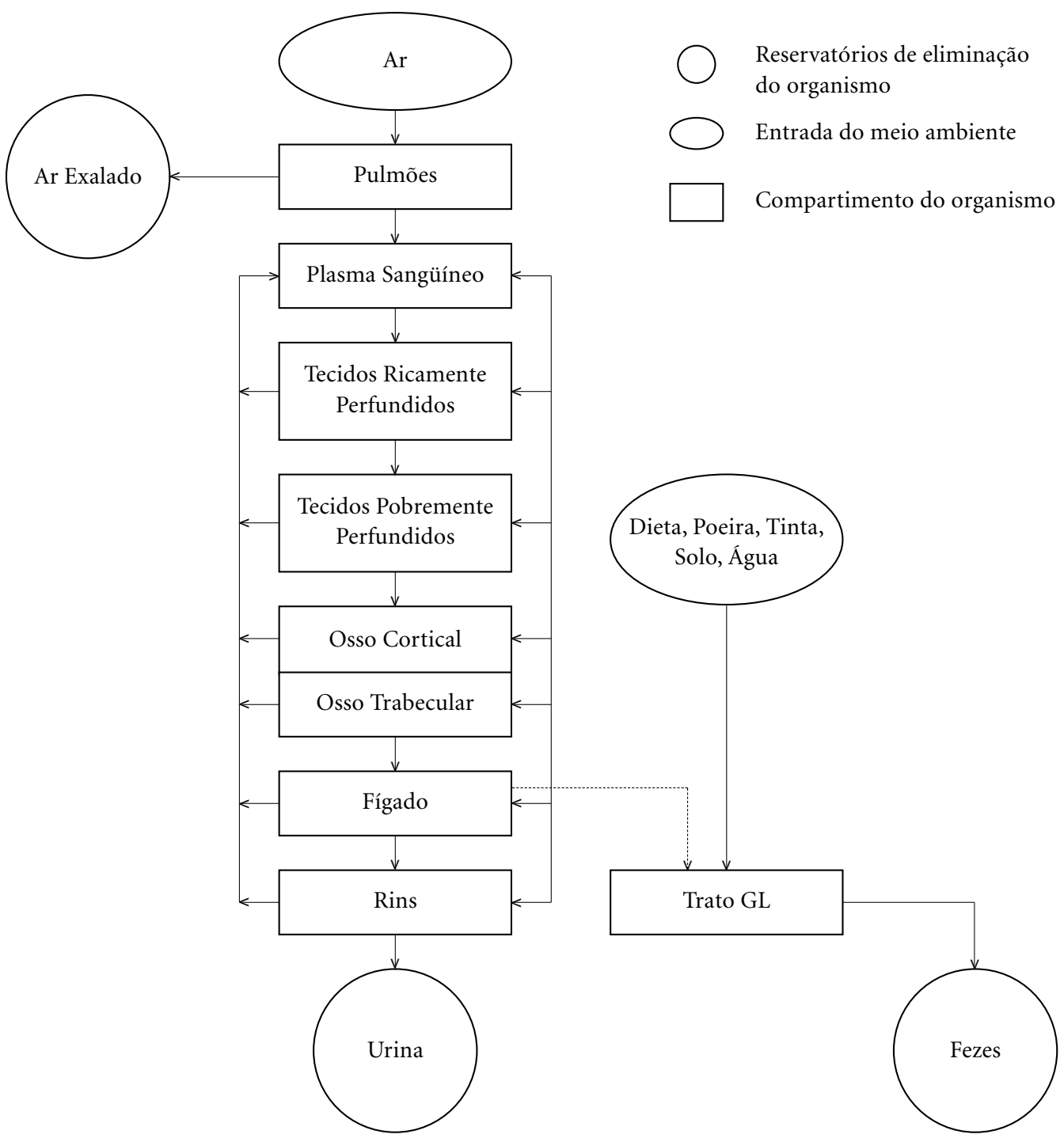

no plasma de modo independente da influência do $\mathrm{Pb}$ eritrocitário. Conseqüentemente, $\mathrm{a}$ superioridade do metal de origem óssea sobre o sangüíneo em predizer algumas formas crônicas de toxicidade pode ser intermediada pela sua influência sobre o $\mathrm{Pb}$ plasmático (Hernandez-Avila, 1998). Assim, uma hipótese alternativa foi proposta para a cinética do $\mathrm{Pb}$, em que fontes endógenas favorecem mais fortemente o compartimento plasma do que as exógenas (Cake et al., 1996). Entretanto, dados obtidos no estudo de Gulson e colaboradores (2000b), com mulheres acompanhadas desde antes da gravidez até 180 dias após o parto, não apóiam esta proposição.

A determinação do chumbo no plasma é uma medida mais exata do que no sangue para avaliação da exposição, especialmente em níveis elevados do metal. O plasma é o componente do sangue que contém o Pb livre para cruzar as membranas celulares e causar seus efeitos tóxicos (Hirata et al., 1995). Os ligantes presentes no plasma, e disponíveis para a ligação com o $\mathrm{Pb}$, ainda não estão bem definidos, 
mas alguns pesquisadores têm sugerido a existência de frações difusíveis de chumbo em equilíbrio com sítios ativos de órgãos-alvo e tecidos moles, e também com as células vermelhas (Goyer, 1991). Sabe-se, entretanto, que o plasma contém uma fração estimada entre $0,2 \%$ a $10 \%$ do $\mathrm{Pb}-\mathrm{S}$, provavelmente ligada à albumina, $\alpha_{2}$-globulina ou como íons livres disponíveis para o transporte para os tecidos. É possível que esta última fração tenha maior importância toxicológica do que o chumbo nos eritrócitos, por estar mais biodisponível para atingir os sítios-alvo da ação toxicológica. As concentrações plasmáticas de chumbo raramente têm sido medidas devido às dificuldades analíticas e, por isso, sua relação com as manifestações tóxicas não está claramente definida (Bergdahl et al., 1997b; Tsaih et al., 1999).

Um estudo longitudinal, realizado com mulheres em idade reprodutiva expostas ambientalmente ao chumbo, encontrou uma variação natural de duas a quatro vezes na partição relativa deste metal entre o sangue total e o plasma, para a faixa de concentração estudada $(2,13$ $39,7 \mu \mathrm{g} . \mathrm{dL}^{-1}$ ) de chumbo em sangue (Smith et al., 2002).

O chumbo presente no sangue é distribuído entre os órgãos, dependendo do gradiente de concentração e da afinidade pelo tecido específico. Níveis mais elevados têm sido encontrados na aorta, fígado e rins. A retenção do chumbo nos tecidos moles se estabiliza na vida adulta e pode decrescer em alguns órgãos com a idade, contudo continua a se acumular nos ossos e na aorta durante toda a vida (Tsalev \& Zaprianov, 1985; Saryan \& Zenz, 1994).

O chumbo atravessa a barreira hemato-encefálica, mas sua concentração no cérebro é baixa. Também penetra na placenta, que pode determinar $57,4 \%$ do nível de $\mathrm{Pb}$ transferido para o feto, e o sangue fetal contém quase que os mesmos níveis do metal que o sangue materno (Nashashibi et al., 1999). O chumbo é mobilizado dos ossos numa taxa acelerada e transferido para o feto, mostrando que esta mobilização contribui significativamente para aumentar os níveis de $\mathrm{Pb}$-S durante a segunda metade da gravidez (Hertz-Picciollo et al., 2000). As concentrações de chumbo no sangue em amostras coletadas do cordão umbilical diminuíram por cerca de 90 dias após o parto, quando então aumentaram até $166 \%$, refletindo os ganhos de chumbo de fontes tais como o esqueleto materno no período de aleitamento (Gulson et al., 1999a).
Durante a sua distribuição, o chumbo segue a via metabólica do cálcio e se acumula nos ossos e dentes. Após examinar a relação entre o chumbo no dente de criança e os níveis de chumbo no osso de adultos jovens, Kim e colaboradores (1996) concluíram que a exposição ao metal na infância pode ser usada para predizer o conteúdo corpóreo até 13 anos depois. Esse grande depósito contribui significativamente para manter os níveis de chumbo no sangue após o término da exposição e também como fonte deste metal para o feto, no período de formação do esqueleto fetal (Gulson et al., 1999c).

Como o chumbo é qualitativamente um análogo biológico do cálcio, sua entrada e liberação do esqueleto são, em parte, controladas por muitos dos mecanismos que regulam a homeostase do mineral. Estes incluem os processos normais de difusão mineral e de troca (aposição/ressorção) (Smith et al., 1996). O Pb armazenado no esqueleto pode ser mobilizado para a circulação nas situações em que normalmente ocorre uma maior mobilização como nos estados fisiológicos e patológicos, que promovem a ressorção óssea (Pires et al., 2001), constituindo assim um mecanismo para a toxicidade tardia. Alguns pesquisadores sugerem que este fato acontece durante a homeostase normal, que ocorre nos períodos de troca óssea acelerada e perda de minerais (Vahter et al., 2002) tais como crescimento rápido de crianças (Sanín et al., 1998), gravidez (Rothenberg et al., 2000), lactação (Gulson et al., 1998b; Tellez-Rojo, 2002) e menopausa (Roberts \& Silbergeld, 1995; Hernandez-Avila et al., 2000). Há suspeitas de que, nos casos de distúrbios no equilíbrio ácido-base, infecções, intervenções cirúrgicas, osteoporose (Silbergeld et al., 1988; Weyermann \& Brenner, 1998), tirotoxicose (Goldman et al., 1994) e terapias com certas drogas (Osterode et al., 2002), o chumbo também possa ser inesperadamente mobilizado, levando à aparição de sintomas tóxicos, mesmo depois de cessada a exposição (Silbergeld, 1993; Saryan \& Zenz, 1994; ATSDR, 1999).

A modelagem apresentada por Rust et al. (1999) sugere que a mobilização do $\mathrm{Pb}$ do osso pode causar impacto no teor deste metal no sangue de crianças pequenas por períodos consideravelmente longos após uma intervenção, explicando o fato, aparentemente contraditório, de que apenas pequenos declínios são observados nas concentrações de $\mathrm{Pb}-\mathrm{S}$, apesar da redução significativa nos níveis de $\mathrm{Pb}$ em poei- 
ra, tinta e solos residenciais encontrados após intervenções.

Gulson e colaboradores (1999b) concluíram que, nos casos em que os níveis de exposição ambiental ao $\mathrm{Pb}$ e a sua ingestão na dieta são baixos, a contribuição dos ossos é a dominante para o $\mathrm{Pb}-\mathrm{S}$ durante a gravidez e lactação. Silbergeld (1991) verificou que o osso é uma fonte potencial de $\mathrm{Pb}$ para o feto e neonato, e que sua cinética é semelhante àquela do Ca ósseo durante os períodos de gravidez e lactação. Estudando um grupo de mulheres pósmenopausa, esta autora também constatou que, durante as gestações (e possíveis lactações), houve mobilização do $\mathrm{Pb}$ do osso, de modo que menor quantidade do metal estava disponível para mobilização durante a desmineralização que ocorre após a menopausa (1991). Symanski e Hertz-Picciotto (1995) chegaram a idêntico resultado, sendo que o acréscimo foi ainda maior nas fumantes. Também concluíram que mulheres com menopausa recente apresentavam concentrações de $\mathrm{Pb}-\mathrm{S}$ mais elevadas do que aquelas cuja menopausa já ocorrera há mais de 4 anos.

Em um estudo realizado com mulheres acima de 65 anos, Muldoon e colaboradores (1994) encontraram associação positiva entre o nível de chumbo no sangue e residência urbana, hábito de fumar, consumo de álcool e tempo após menopausa, enquanto que índice de massa corporal, amamentação, terapia de reposição hormonal em curso, moderada atividade física e ingestão de cálcio estavam inversamente correlacionadas. A reposição hormonal foi prescrita para 33 das 55 mulheres com média de 3,5 anos pós-menopausa recrutadas para a pesquisa e, após 4 anos de terapia, o conteúdo de chumbo foi medido nos ossos e também foram determinadas as concentrações deste metal no soro, sangue total e urina. $\mathrm{O}$ teor de chumbo no osso cortical das mulheres sem tratamento foi significativamente menor quando comparado com o daquelas com reposição, enquanto que não houve diferenças para as concentrações do metal medidas no osso trabecular, sangue total, soro ou urina entre os grupos (Webber et al., 1995).

A macrodistribuição do chumbo no sistema ósseo não é uniforme e é claramente dependente do tipo de osso (cortical ou trabecular), idade e, em menor grau, do gênero (Aufderheide e Wittmers, 1992). Silbergeld (1991) sugere que as interações entre o chumbo e o cálcio são os meios moleculares fundamentais para a toxicidade do chumbo em mulheres ao longo de suas vidas.

Convencionalmente, considera-se que o osso total seja formado por cerca de $20 \%$ de osso trabecular e $80 \%$ de osso cortical (O'Flaherty, 1998). A concentração de cálcio no tecido ósseo é de aproximadamente $22,5 \%$ no osso cortical puro, enquanto que $7,4 \%$ do teor deste mineral é encontrado no trabecular. A taxa de renovação do osso trabecular é descrita como sendo de 3 a 10 vezes aquela do osso cortical, o que se reflete na taxa de mobilização do $\mathrm{Pb}$, relatada ser mais rápida no trabecular do que no osso cortical (Nilsson et al., 1991). Assim, há, pelo menos, dois reservatórios diferentes de $\mathrm{Pb}$ no esqueleto. $\mathrm{O}$ compartimento lábil (trabecular), que rapidamente troca o metal com o sangue, é responsável pela manutenção de um equilíbrio entre o osso e o tecido mole ou sangue, enquanto que no outro (cortical), o chumbo é essencialmente inerte, com um tempo de meia-vida de várias décadas. Mesmo assim, este reservatório inerte representa um risco especial, já que é uma fonte endógena potencial de chumbo (ATSDR, 1999). Como o centro dos ossos longos possui estrutura cortical, o depósito neste tipo de osso mostra gradientes de concentração relacionados com o tempo de fixação do chumbo e com o nível de exposição (O’Flaherty, 1998).

O osso trabecular parecer ser uma importante fonte do chumbo reabsorvido quando há redução da exposição, como no caso da remoção do ambiente de trabalho por motivos médicos ou aposentadoria. O metal pode ser inesperadamente mobilizado com aparição de sintomas tóxicos, mesmo após cessada a exposição (IPCS, 1995). Hernandez-Avila e colaboradores (1996), utilizando a patela como representativa do osso trabecular, mostraram a contribuição significativa deste tipo de osso para o chumbo do sangue durante a lactação. Este estudo também evidencia que o consumo de elevados teores de cálcio reduz o acúmulo de chumbo neste tipo de osso, o que não foi observado para a tíbia (representativa do osso cortical).

Estudos realizados com trabalhadores em atividade mostraram que a concentração de chumbo na tíbia (representativo do osso cortical) aumentou consistentemente como função da intensidade e duração da exposição, já a concentração do chumbo no calcâneo (representativo do osso trabecular) foi fortemente dependente apenas da intensidade. $O$ fato indicou que o tempo de meia-vida do chumbo 
no calcâneo foi menor do que o período de exposição (sete a oito anos). Enquanto que os resultados encontrados para trabalhadores aposentados demonstraram claramente que a exposição endógena ao chumbo, proveniente da mobilização do conteúdo ósseo acumulado durante toda a vida laboral, pode facilmente se constituir na contribuição predominante para concentrações sistemáticas de chumbo após cessada a exposição ocupacional (Erkkila et al., 1992).

\section{Eliminação}

O chumbo é excretado por várias rotas, porém só a excreção renal e a gastrointestinal são de importância prática. A quantidade excretada, independente da rota, é afetada pela idade, características da exposição e dependente da espécie. A comparação dos dados sobre a cinética do chumbo em adultos e crianças mostra que, aparentemente, estas últimas parecem ter uma taxa total de excreção menor. Crianças até dois anos de idade retêm $34 \%$ da quantidade total de chumbo absorvido, enquanto que esta retenção é de apenas $1 \%$ nos adultos. Num estudo sobre a relação entre o nível de exposição e a taxa de excreção do chumbo foi encontrado que a eliminação renal, para valores de chumbo no sangue entre 25 e $80 \mu \mathrm{g} . \mathrm{dL}^{-1}$, aumenta numa taxa semelhante àquela de aumento do chumbo no plasma (IPCS, 1995).

A excreção gastrointestinal acontece por secreção de várias glândulas, entre elas a pancreática, e por excreção biliar, possivelmente na forma de um complexo chumbo-glutationa. Uma fração desconhecida, porém provavelmente importante, de chumbo excretada desta forma é reabsorvida. O chumbo da dieta ou, até mesmo, aquele engolido nas partículas de ar e não absorvido pelo trato gastrointestinal é eliminado nas fezes, que contêm elevado teor do metal, em torno de $90 \%$ do total excretado. A maior parte do chumbo encontrado nas fezes é constituída principalmente pelo metal não absorvido, que passa pelo trato gastrointestinal. O restante é derivado da diferença entre a excreção gastrointestinal total e a reabsorção. A distinção entre o chumbo não absorvido e o excretado após absorção tem sido um sério obstáculo para o entendimento da verdadeira excreção gastrointestinal e, por esta razão, existem opiniões conflitantes quanto à sua importância (Saryan e Zenz, 1994).
A excreção do chumbo absorvido nas fezes é cerca de metade da urinária em baixas exposições e, provavelmente, menor nos níveis mais elevados. Entretanto, um experimento realizado com dois voluntários, que receberam $212 \mathrm{~Pb}$ por via intravenosa, mostrou a ausência de $\mathrm{Pb}$ nas fezes durante as primeiras 24 horas, enquanto que a urina continha $4,42 \%$ da dose administrada. No entanto, no segundo dia, esses valores foram $1,5 \%$ e $1,42 \%$, respectivamente, indicando que a rota fecal pode contribuir tanto quanto a urinária para a excreção total (Saryan e Zenz, 1994).

Os mecanismos exatos de excreção do chumbo para a urina não foram determinados devido a dificuldades associadas com a medição do metal ultrafiltrável no plasma e, por conseqüência, em medir a sua taxa de filtração glomerular (ATSDR, 1999). A excreção renal acontece predominantemente, talvez exclusivamente, por filtração glomerular, e provavelmente é seguida por reabsorção tubular parcial. No entanto, o papel da reabsorção tubular não está completamente elucidado. A taxa de excreção urinária do chumbo é afetada pelo fluxo urinário, com um decréscimo durante a noite. Uma vez que a concentração do chumbo na urina reflete exposição atual, a medida deste parâmetro tem sido aplicada em saúde ocupacional como teste de exposição (Skerfving, 1993).

Tsaih e colaboradores (1999) examinaram as relações entre os níveis de chumbo no sangue total corrigido pelo hematócrito e os teores do metal nos ossos trabecular e cortical, e urina de 24 horas de 71 homens de meia idade e idade avançada, sem conhecida exposição ocupacional ao chumbo. Como o $\mathrm{Pb}$ na urina $(\mathrm{Pb}-$ $\mathrm{U})$ se origina do $\mathrm{Pb}$ plasmático filtrado nos glomérulos, concluíram que o $\mathrm{Pb}$ urinário, ajustado para a taxa de filtração glomerular, serve como substituto para a determinação de chumbo no plasma ( $\mathrm{Pb}-\mathrm{P})$, útil na avaliação dos danos em populações expostas ambientalmente. Conclusão idêntica a que Hirata e seus colegas chegaram, após encontrarem uma correlação significativa mais forte entre $\mathrm{Pb}-\mathrm{P} \mathrm{e} \mathrm{Pb}$ $\mathrm{U}$ do que entre $\mathrm{Pb}-\mathrm{S}$ e $\mathrm{Pb}-\mathrm{U}$, em amostras de trabalhadores expostos a baixos e elevados níveis do metal (1995).

Gulson et al. (2000a) concluíram que não houve diferença nas taxas de excreção durante os trimestres da gravidez e entre os períodos de gestação e pós-parto em amostras de urinas pontuais e de 24 horas de treze mulheres. Em 
outro estudo, $\mathrm{o} \mathrm{Pb}$ dos tecidos foi identificado isotopicamente na urina e os autores concluíram que a maior excreção em relação à ingestão, considerando desprezíveis as entradas de chumbo provenientes do ar, solo e poeira, refletia a mobilização deste metal dos tecidos dos recém-nascidos, associada com a rápida renovação dos ossos neste estágio da vida (Gulson, 2001b).

Nas suas investigações, Tell e colaboradores (1992) encontraram uma correlação muito próxima entre o nível de chumbo no sangue de trabalhadores expostos ocupacionalmente e sua excreção urinária, 24 horas após injeção intravenosa com ácido etilenodiamino tetracético (EDTA). Concluíram, então, que o metal disponível para quelação reflete principalmente o seu depósito no sangue e tecidos moles, que é só parcialmente dependente do teor de chumbo dos ossos, onde é encontrada a maior parte do conteúdo corpóreo total. Resultados semelhantes encontraram Gerhardsson e colegas (1999), usando ácido 2,3 meso-dimercaptosuccínico (DMSA) como agente quelante, em que o chumbo quelado excretado na amostra de urina de 24 horas se correlacionou bem com a excreção na porção de 6 horas. A amostra de urina de 6 horas mostrou uma relação não linear com chumbo no sangue e relações lineares com Pb-plasma e chumbo na urina da manhã. Assim, puderam inferir que o metal quelado com DMSA reflete principalmente a sua concentração no sangue, tecidos moles e, possivelmente, também osso trabecular, não sendo, por isso, um bom indicador do conteúdo corpóreo total e de exposição crônica. Para tais estimativas, o teor de chumbo no osso cortical é mais correto, já que contém a maior fração de $\mathrm{Pb}$ acumulada no organismo ao longo do tempo. Além disso, o teste de mobilização não deu melhor informação do que as medidas dos níveis de $\mathrm{Pb}$ no sangue, plasma, ou urina sem quelação. Hu e colaboradores (1991) usaram a fluorescência de raio X-K para medir a concentração de $\mathrm{Pb}$ na tíbia e patela de sujeitos expostos ocupacionalmente. Os dados encontrados sugerem que os teores de $\mathrm{Pb}$ nos ossos correspondem linearmente ao $\mathrm{Pb}$ urinário após teste de mobilização com EDTA, e que uma terapêutica de quelação entre 3 e 5 dias não é suficiente para diminuir os níveis do metal nos ossos.

Além de o chumbo absorvido ser excretado principalmente pela urina $(75-80 \%)$ e cerca de $15 \%$ pelas fezes, através da bílis e secreção do trato gastrointestinal, outras possíveis rotas de eliminação são suor, descamação cutânea, cabelo e unhas (<8\%) (Tsalev e Zaprianov, 1985). Uma via especial de excreção do chumbo endógeno é através do leite materno. Embora este tipo de eliminação tenha pouca importância do ponto de vista de liberação do organismo, pode representar um risco para o lactente, já que existe uma correlação entre a concentração do chumbo no sangue e o leite. A maioria dos estudos relata que os níveis de chumbo no leite materno variam entre $10 \%$ e $30 \%$ daqueles do sangue materno, sendo geralmente na ordem de $0,21 \mu \mathrm{g} . \mathrm{dL}^{-1}$, e possivelmente mais elevados no colostro do que no leite maduro (Skerfving, 1993). Os teores de chumbo no leite materno são similares àqueles no plasma, e as crianças estarão sob risco se a mãe estiver exposta a elevadas concentrações do metal, qualquer que seja a fonte, endógena ou exógena, concluíram Gulson et al. (1998a). Baixos níveis de chumbo têm sido encontrados no sêmen de homens sem exposição específica. Parece que uma porção significativa se origina da próstata ou da vesícula seminal. Além disso, trabalhadores expostos ao chumbo têm concentrações aumentadas do metal no fluido seminal, que são, aproximadamente, um décimo daquelas no sangue (Skerfving, 1993).

O organismo acumula chumbo durante toda a vida e o libera de forma extremamente lenta, devido à sua grande afinidade pelo sistema ósseo. Conseqüentemente, após uma única exposição, o nível de chumbo no sangue de uma pessoa pode retornar ao normal e, no entanto, o conteúdo corpóreo total pode ainda ser elevado. Mesmo doses pequenas, por um tempo determinado, podem causar intoxicação. Assim, grandes exposições agudas não precisam ocorrer para que uma intoxicação por chumbo se desenvolva. O conteúdo total de chumbo no corpo é que está relacionado com o risco de efeitos adversos (ATSDR, 1992).

\section{Conclusão}

Apesar de a absorção, distribuição e eliminação do chumbo virem sendo exaustivamente estudadas em animais e homens nos últimos tempos, alguns dos mecanismos controladores destes processos fisiológicos continuam desconhecidos.

Os mecanismos exatos pelos quais o chumbo é absorvido pelo intestino são desconhecidos, embora numerosas observações sugiram a 
existência de um mecanismo de absorção saturável ou algum outro processo que limite a entrada do chumbo em humanos, enquanto outras propõem um efeito de dose sobre a absorção ou algum outro aspecto da biocinética do chumbo. Sabe-se que a absorção intestinal do chumbo é influenciada pela dieta e pelo estado nutricional de cálcio e ferro, porém possíveis mecanismos têm sido propostos para estas interações.

O chumbo no sangue se encontra no plasma e, em maior parte, associado às células vermelhas. Porém, existem controvérsias quanto ao transporte do $\mathrm{Pb}$ pelo componente plasma do sangue, que pode ser mais influenciado pelos níveis deste metal proveniente do osso, uma fonte significativa de chumbo para o plasma. Desta forma, o chumbo plasmático poderia intermediar a predição de algumas formas crônicas de toxicidade. Entretanto, nem todas as espécies de chumbo existentes no plasma, bem como nos eritrócitos, são conhecidas. Portanto, a especiação, ou seja, a caracterização dos constituintes que se ligam e/ou que transportam o elemento de interesse é fundamental para o total entendimento do metabolismo, elucidação dos mecanismos de toxicidade, melhor compreensão do modo de entrada ou de distribuição do metal na célula, ou ainda para o estudo das interações do chumbo com as proteínas e deposição nos tecidos.

Todo chumbo não absorvido pelo trato gastrointestinal é eliminado pelas fezes, inclusive aquele proveniente do ar, que foi engolido e não absorvido. $O$ metal não retido no organismo é eliminado pelos rins ou excretado pela bile para o trato gastrointestinal. A excreção pela urina ocorre através de processos de filtração glomerular, provavelmente seguida de reabsorção tubular parcial, e pode ser afetada pelo fluxo urinário, enquanto que a sua elimi- nação pela bile se dá provavelmente na forma de um complexo chumbo-glutationa. No entanto, como acontece com outros processos fisiológicos, os mecanismos precisos de excreção do chumbo pela urina bem como pelas fezes ainda não foram totalmente elucidados.

Como se pode perceber, muitas pesquisas ainda precisam ser desenvolvidas na tentativa de esclarecer os reais ligantes e mecanismos de ação do chumbo, que permanecem obscuros. Estudos com modelos farmacocinéticos fisiológicos podem oferecer uma contribuição importante quanto a esses aspectos da cinética do chumbo, se não os elucidando completamente, direcionando futuras pesquisas a partir de modelos desenvolvidos em bases anatômicas e fisiológicas, ajustáveis a escalas alométricas e de alto grau de correlação.

Assim, o conhecimento da cinética do chumbo é de extrema importância para maior compreensão da toxicidade do metal, uma vez que os riscos de efeitos adversos à saúde estão relacionados com o conteúdo corpóreo total do chumbo. Por isso, qualquer pesquisa que tenha por finalidade avaliar a exposição a este elemento com mais especificidade e sensibilidade estará contribuindo para o aprofundamento da questão e para a proposição de intervenções mais adequadas. A percepção da série de eventos entre a exposição ao chumbo e as alterações biológicas, que levam aos efeitos adversos, é fundamental para o desenvolvimento de métodos relevantes de monitoramento biológico. Especificamente, os estudos de novos modelos biológicos tais como a especiação e o polimorfismo da enzima ALAD, que além de fornecer base para melhor compreensão dos mecanismos de ação do chumbo no organismo, serão de grande utilidade na avaliação dos efeitos tóxicos observados em populações expostas ao metal em questão. 


\section{Referências bibliográficas}

ATSDR (Agency for Toxic Substances and Disease Registry) 1992. Case studies in environmental medicinelead toxicity. US Department of Health and Human Services, Public Health Service, Atlanta.

ATSDR (Agency for Toxic Substances and Disease Registry) 1999. Toxicological profile for lead. US Department of Health and Human Services, Public Health Service, Atlanta.

Aufderheide AC \& Wittmers LEJr 1992. Selected aspects of the spatial distribution of lead in bone. Neurotoxicology 13(4):809-819.

Baghurst PA et al. 1987. Determinants of blood lead concentrations of pregnant women living in Port Pirie and surrounding areas. Medical Journal of Australia 146(2):69-73.

Bergdahl IA et al. 1997a. Lead binding to delta-aminolevulinic acid dehydratase (ALAD) in human erythrocytes. Pharmacology \& Toxicology 81(4):153-158.

Bergdahl IA, Schutz A, Gerhardsson L, Jensen A \& Skerfving S 1997b. Lead concentrations in human plasma, urine and whole blood. Scandinavian Journal of Work and Environmental Health 23(5):359-363.

Bergdahl IA, Sheveleva M, Schultz A, Artamonova VG \& Skerfving S 1998. Plasma and blood lead in humans: capacity-limited binding to delta-aminolevulinic acid dehydratase and other lead-binding components. Toxicological Sciences 46(2):247-253.

Bourgoin BP, Evans DR, Cornett JR, Lingard SM \& Quattrone AJ 1993. Lead content in 70 brands of dietary calcium supplements. American Journal of Public Health 83(8):1155-1160.

Cake KM et al. 1996. Partition of circulating lead between serum and red cells is different for internal and external sources of lead. American Journal of Industrial Medicine 29(5):440-445.

Cheng Y et al. 1998. Relation of nutrition to bone lead and blood lead levels in middle-aged to elderly men. The normative aging study. American Journal of Epidemiology 147(12):1162-1174.

Erkkila J et al. 1992. In vivo measurements of lead in bone at four anatomical sites: long term occupational and consequent endogenous exposure. British Journal of Industrial Medicine 49(9):641-644.

Fleming DE, Chettle DR, Webber CE \& O'Flaherty EJ 1999. The O'Flaherty model of lead kinetics: an evaluation using data from a lead smelter population. Toxicology and Applied Pharmacology 161(1):100-109.

Fleming DEB et al. 1997. Accumulated body burden and endogenous release of lead in employees of a lead smelter. Environmental Health Perspectives 105(2): 224-233.

Fullmer CS 1995. Dietary calcium levels and treatment interval determine the effects of lead ingestion on plasma 1,25-dihydroxyvitamin D concentration in chicks. The Journal of Nutrition 125(5):1328-1333.

Fullmer CS 1997. Lead-calcium interactions: Involvement of 1,25-dihydroxyvitamin D. Environmental Research 72(1):45-55.

Gerhardsson L, Borjesson J, Mattsson S, Schutz A \& Skerfving S 1999. Chelated lead in relation to lead in bone and ALAD genotype. Environmental Research 80(4):389-398.

Goldman RH, White R, Kales SN \& Hu H 1994. Lead poi- soning from mobilization of bone stores during thyrotoxicosis. American Journal of Industrial Medicine 25(3):417-424.

Goyer RA 1991. Toxic effects of metals - Lead, pp. 639-646. In Mary O. Amdur, John Dull \& Curtis D. Klaassen (eds.). Casareh and Doull's toxicology - the basic science of poisons. Editora Pergamon Press, USA.

Gulson BL et al. 1995. Contribution of tissue lead to blood lead in adult female subjects based on stable lead isotope methods. Journal of Laboratory and Clinical Medicine 125(6):703-712.

Gulson BL et al. 1997a. Pregnancy increases mobilization of lead from maternal skeleton. Journal of Laboratory and Clinical Medicine 130(1):51-62.

Gulson BL et al. 1997b. Dietary lead intakes for mother/ child pairs and relevance to phamacokinetic models. Environmental Health Perspectives 105(12):1334-1342.

Gulson BL et al. 1998a. Relationships of lead in breast milk to lead in blood, urine and diet of infant and mother. Environmental Health Perspectives 106(10):667-674.

Gulson BL et al. 1998b. Mobilization of lead from the skeleton during the postnatal period is larger than during pregnancy. Journal of Laboratory and Clinical Medicine 131(4):324-329.

Gulson BL et al. 1999a. Comparison of rates of exchange of lead in the blood of newly born infants and their mothers with lead from their current environment. Journal of Laboratory and Clinical Medicine 133(2): 171-178.

Gulson BL et al. 1999b. Impact of diet on lead in blood and urine in female adults and relevance to mobilization of lead from bone stores. Environmental Health Perspectives 107(4):257-263.

Gulson BL et al. 1999c. Estimation of cumulative lead releases (lead flux) from the maternal skeleton during pregnancy and lactation. Journal of Laboratory and Clinical Medicine 134(6):631-640.

Gulson BL, Mizon KJ, Korsch MJ \& Donnelly JB 2000a. Urinary excretion of lead during pregnancy and postpartum. Science of Total Environment 262(1-2):49-55.

Gulson BL et al. 2000b. Urinary lead isotopes during pregnancy and postpartum indicate no preferential partitioning of endogenous lead into plasma. Journal of Laboratory and Clinical Medicine 136(3):236-242.

Gulson BL, Mizon KJ, Palmer JM, Korsch MJ \& Taylor AJ 2001a. Contribution of lead from calcium supplements to blood lead. Environmental Health Perspectives 109(3):283-288.

Gulson BL et al. 2001b. Longitudinal study of daily intake and excretion of lead in newly born infants. Environmental Research 85(3):232-245.

Han SG et al. 2000. Effects of lead exposure before pregnancy and dietary calcium during pregnancy on fetal development and lead accumulation. Environmental Health Perspectives 108(6):527-531.

Hernandez-Avila M et al. 1996. Dietary and environmental determinants of blood and bone lead levels in lacting postpartum women living in Mexico City. Environmental Health Perspectives 104(10):1076-1082.

Hernandez-Avila M, Smith D, Meneses F, Sanin LH \& Hu H 1998. The influence of bone and blood lead on plasma lead levels in environmentally exposed adults. Environmental Health Perspectives 106(8):473-477. 
Hernandez-Avila M et al. 2000. Determinants of blood lead Levels across the menopausal transition. Archieves of Environmental Health 55(5):355-360.

Hertz-Picciotto I et al. 2000. Patterns and determinants of blood lead during pregnancy. American Journal of Epidemiology 152(9):829-837.

Hirata M, Yoshida T, Miyajima K, Kosaka H \& Tabuchi T 1995. Correlation beteween lead in plasma and other indicators of lead exposure among lead-exposed workers. International Archieves of Occupational and Environmental Health 68:58-63.

Hryhorczuk DO et al. 1985. Elimination kinetics of blood lead in workers with chronic lead intoxication. American Journal of Industrial Medicine 8(1):33-42.

Hu H, Pepper L \& Goldman R 1991. Effect of repeated occupational exposure to lead, cessation of exposure, and chelation on levels of lead in bone. American Journal of Industrial Medicine 20(6):723-735.

$\mathrm{Hu} \mathrm{H} \mathrm{1998.} \mathrm{Bone} \mathrm{lead} \mathrm{as} \mathrm{a} \mathrm{new} \mathrm{biologic} \mathrm{marker} \mathrm{of} \mathrm{lead}$ dose: recent findings and implications for public health. Environmental Health Perspectives 106(Suppl. 4):961-967.

IPCS (International Programme on Chemical Safety) 1995. Environmental Health Criteria 165 - Inorganic Lead. World Health Organization, Geneva, Suíça.

Johnson MA 2001. High calcium intake blunts pregnancy-induced increases in maternal blood lead. Nutrition Review 59(5):152-156.

Kim R, Hu H, Rotnitzky A, Bellinger D \& Needleman H 1996. Longitudinal relationship between dentin lead levels in childhood and bone lead levels in young adulthood. Archieves of Environmental Health 51(5): 375-382.

Kostial K et al. 1991. Dietary calcium and blood lead levels in women. Biological Trace Element Research 28(3): 181-185.

Kristal-Boneh E et al. 1998. Effect of dietary calcium on blood lead concentration in occupationally exposed and nonexposed workers. American Journal of Industrial Medicine 34(5):512-516.

Morris C, McCaron DA \& Bennett WM 1990. Low-level lead exposure, blood pressure, and calcium metabolism. American Journal of Kidney Disease 15(6):568574.

Muldoon SB, Cauley JA, Kuller LH, Scott J \& Rohay J 1994. Life-style and sociodemographic factors as determinants of blood lead levels in elderly women. American Journal of Epidemiology 139(6):599-608.

Nashashibi N, Cardamakis E, Bolbos G \& Tzingounis V 1999. Investigation of kinetic of lead during pregnancy and lactation. Gynecologic and Obstetric Investigation 48(3):158-162.

Nilsson U et al. 1991. Kinetics of lead in bone and blood after end of occupational exposure. Pharmacology of Toxicology 69(6):477-484.

O’Flaherty EJ 1995. Physiologically based models for bone-seeking elements - V. Lead absorption and deposition in childhood. Toxicology and Applied Pharmacology 131(2):297-308.

O'Flaherty EJ 1998. A physiologically based kinetics model for lead in children and adults. Environmental Health Perspectives 106(Suppl.6):1495-1503.

Osterode W, Zettinig G, Potzi C \& Manner G 2002. Increased lead excretion in hypothyroid patients after levothyroxine medication. Journal of Toxicology and Environmental Health 65(9):649-654.
Pires BJ, Bezerra FF, Laboissiere FP, Miekeley N \& Donangelo CM 2001. Lead levels in erythrocytes and biomarkers of bone turnover in pregnant and lactating women with marginal calcium intakes. Nutrition Research 21(6):831-841.

Rabinowitz MB 1991. Toxicokinetics of bone lead. Environmental Health Perspectives 91:33-37.

Rabinowitz MB, Wetherill GW \& Kopple JD 1976. Kinetic analysis of lead metabolism in healthy humans. The Journal of Clinical Investigation 58(2):260-270.

Robert JS \& Silbergeld EK 1995. Pregnancy, lactation, and menopause - how physiology and gender affect the toxicity of chemicals. Mount Sinai Journal of Medicine 62(5):343-355.

Ross EA, Szabo NJ \& Tebbett IR 2000. Lead content of calcium supplements. Journal of American Medicine Association 284(11):1425-1429.

Rothenberg SJ et al. 1994. Changes in serial blood lead levels during pregnancy. Environmental Health Perspectives 102(10):876-880.

Rothenberg SJ et al. 2000. Maternal bone lead contribution to blood lead during and after pregnancy. Environmental Research 82(1):81-90.

Rust SW, Burgoon, DA, Niemuth NA \& Schultz BD 1999. Influence of bone-lead stores on the observed effectiveness of lead hazard intervention. Environmental Research 81(3):175-184.

Sanín LH, Cossío TG, Romieu I \& Avila MH 1998. Acumulación de plomo en hueso y sus efectos en la salud. Salud Pública de México 40(4):359-368.

Sargent JD, Dalton MA, O'Connor GT, Olmstead EM \& Klein RZ 1999. Randomized trial of calcium glycerophosphate-supplemented infant formula to prevent lead absorption. The American Journal of Clinical Nutrition 69(6):1224-1230.

Saryan LA \& Zenz C 1994. Lead and its compounds, pp. 506-541. In C Zenz, OB Dickerson \& EP Horvath (eds.). Occupational medicine. Editora Mosby-Year Book, Inc., EUA.

Scelfo GM \& Flegal AR 2000. Lead in calcium supplements. Environmental Health Perspectives 108(4):309-319.

Silbergeld EK 1991. Lead in bone: implications for toxicology during pregnancy and lactation. Environmental Health Perspectives 91:63-70.

Silbergeld EK, Schwartz J \& Mahaffey K 1988. Lead and osteoporosis: mobilization of lead from bone in postmenopausal women. Environmental Research 47(1): 79-94.

Silbergeld EK et al. 1993. Lead in bone - storage site, exposure source, and target organ. Neurotoxicology 14(23):225-236.

Skerfving S 1993. Inorganic Lead, pp. 125-238. In BB Lundberg (edit.). Criteria documents from the Nordic Expert Group. International Labour Organisation, Estocolmo.

Smith RD, Osterloh JD \& Flegal AR 1996. Use of endogenous, stable lead isotopes to determine release of lead from skeleton. Environmental Health Perspectives 104(1):60-66.

Smith D, Hernandez-Avila M, Tellez-Rojo MM, Mercado A \& Hu H 2002. The relationship between lead in plasma and whole blood in women. Environmental Health Perspectives 110(3):263-268.

Symanski E \& Hertz-Picciotto I 1995. Blood lead levels in relation to menopause, smoking, and pregnancy history. American Journal of Epidemiology 141(11):1047-1058. 
Tell I et al. 1992. Chelated lead and bone lead. Scandinavian Journal of Work and Environmental Health 18(2):113-119.

Tellez-Rojo MM et al. 2002. Impact of breastfeeding on the mobilization of lead from bone. American Journal of Epidemiology 155(5):420-428.

Tsaih SW et al. 1999. The independent contribution of bone and erythrocyte lead to urinary lead among middle-aged and elderly men: the normative aging study. Environmental Health Perspectives 107(5):391-396.

Tsalev DL \& Zaprianov ZK 1985. Lead, pp. 137-150. In Atomic absorption spectrometry in occupational and environmental health practice. CRC Press, Flórida.

Vahter M, Berglund M, Akesson A \& Liden C 2002. Metals and women's health. Environmental Research 88(3):145-55.
Webber CE et al. 1995. Hormone replacement therapy may reduce the return of endogenous lead from bone to the circulation. Environmental Health Perspectives 103(12):1150-1153.

Weyermann M \& Brenner H 1998. Factors affecting bone demineralization and blood lead levels of postmenopausal women - a population-based study from Germany. Environmental Research 76(1):19-25.

WHO (World Health Organization) 1996. Biological monitoring of chemical exposure in the workplace - Guidelines. World Health Organization, Geneva, Suíça, vol. 1.

Xie Y, Chiba M, Shinohara A, Watanabe H \& Inaba Y 1998. Studies on lead-binding protein and interaction between lead and selenium in the human erythrocytes. Industrial Health 36:234-239.

Artigo apresentado em 20/12/2002

Aprovado em 4/5/2003

Versão final apresentada em 3/6/2003 